\title{
Il mondo nuovo
}

\section{Brave new world}

\author{
L. Monge \\ ${ }^{1}$ Editor in chief JAMD - The journal of AMD. \\ Corresponding author: amd-to.monge@alma.it
}

Qualche giorno fa ho ricevuto una telefonata da una cara collega che non sentivo dall'era pre-Covid; un affettuoso saluto e prima del consulto, che era il motivo della telefonata, una battuta: "ma ti sei adattato al nuovo mondo? io no..., no, faccio molta fatica" e poi una serie di considerazioni sull'isolamento, sulla mancanza del contatto umano, sulla comunicazione affidata alle macchine. La crisi accelera la fine di ogni socialità, non si parla di distanziamento fisico, ma di distanziamento sociale! Sarà perché il mondo in questa fase postpandemia è diventato un mondo, sì globale, ma fatto di un insieme di individui, al massimo di nuclei familiari, ognuno dei quali impegnato a difendere il proprio spazio?

Una narrazione di sapore quasi distopico che mi ha fatto riflettere, e mi sono trovato a pensare alla possibilità di una storia diversa, positiva, che veda in una comunità ritrovata, percepita di fronte all'emergenza come rinnovata, solidale, una nuova consapevolezza anche per la difesa del pianeta... e poi, forse, ora che anche "l'Europa s'è desta".

Probabilmente entrambe le storie hanno fondamento; lascio a voi la scelta - tra le due - di quale sia quella che si imporrà diventando la trama del romanzo del nostro futuro... ma vorrei credere "all'invincibile aspetto comunitario e solidale dell'umano, che l'oggettività della scienza e della tecnica consente di far emergere in tutta la sua evidenza"*

Citation L. Monge (2020) II mondo nuovo. JAMD Vol. 23/2

DOI 10.36171/jamd 20.23.2.1

Editor Luca Monge, Associazione Medici Diabetologi, Italy

Published July, 2020

Copyright (c) 2020 Monge. This is an open access article edited by $\underline{A M D}$, published by Idelson Gnocchi, distributed under the terms of the Creative Commons Attribution $\mathrm{Li}$ cense, which permits unrestricted use, distribution, and reproduction in any medium, provided the original author and source are credited.

Data Availability Statement All relevant data are within the paper and its Supporting Information files.

Funding The Author received no specific funding for this work.

Tutti questi segni li leggiamo anche in questo numero di JAMD e non posso non partire appunto da una narrazione, quella di Alberto De Micheli, Stefano Parini e Maria Chantal Ponziani, il racconto di chi, medico, è stato malato e ha vissuto il ruolo di paziente. Una riflessione intensa, originale, profonda, sincera che ha già raccolto nella sua anteprima web i commenti positivi di molti colleghi. L'auspicio che ne ricaviamo è che "più consci di essere precari e fragili" potremo lavorare insieme affinché la medicina che verrà sia "più umile e concreta" e più attenta e rispettosa della prevenzione.

Il Covid-19 ha lasciato altri segni.

Due prodotti congiunti AMD-SID: il primo è un progetto promosso dal gruppo Psicologia e diabete che offre la consulenza di psicologi per "gestire il vissuto psicologico dei pazienti affetti da diabete [...] e supportare la gestione della complessità a livello emotivo del

* Cito da: A. Schiavone, Progresso. Il Mulino, Bologna 2020. 
diabetologo" e il secondo un PDTA per lo screening e la gestione del diabete in gravidanza durante la pandemia Covid-19 a cura del gruppo di studio Diabete e gravidanza.

In questo numero le glifozine hanno un ruolo centrale con lo studio osservazionale SIDECAR presentato da Valentina De Mori e colleghi che analizza nel real world la loro efficacia e l'incidenza di effetti avversi. Il dato che mi ha colpito di più è l'elevata sospensione precoce dei farmaci, addirittura un 38\% complessivo, nel 14\% per inefficacia e nel 15\% per comparsa d'infezioni del tratto genito-urinario, un dato che fa riflettere sul mancato beneficio cardiovascolare per i nostri pazienti. Nascono spontanee alcune domande: perché le glifozine, come peraltro suggerito dalle linee guida, non sono utilizzate indipendentemente dall'effetto sulla glicemia? e l'associazione con i DPP-4 può essere un'opzione da utilizzare in prima battuta per ridurre l'incidenza dei GUTIs?

Particolarmente utile a questo proposito è la rassegna, a primo nome Tuccinardi, sulla terapia dello scompenso cardiaco, rassegna che ribadisce la centralità delle glifozine nel trattamento di questa sindrome e quindi la necessità di un'ottimizzazione del loro utilizzo.

Una particolare attenzione va posta all'articolo di Riccio che nella sua riflessione su dove va l'educazione terapeutica, dopo una puntualizzazione storica sul ruolo dell'ET nella cura del diabete, sostiene convintamente un'evoluzione verso un'educazione terapeutica prevalentemente in senso digitale. L'articolo entra in un dialogo serrato con un commento dell'attuale coordinatore del Gised, Emanuele Fraticelli.

Personalmente penso che come nel caso dello smart-working, dei webinar o della telemedicina, la tecnologia web ci possa essere di grande aiuto (e di fatto si è dimostrata risolutiva in condizioni di emergenza come quelle determinatesi nel corso dell'epidemia), ma che la prossimità fisica (ora sarà anche rischiosa!) abbia un plus incommensurabile in termini di umanità, creatività, dialogo, volontà. Faccio mia, a tal proposito, la chiusura dell'articolo di Fraticelli: "La Rete è centrata sulla malattia. L'educatore è focalizzato sulla persona."

L'approccio all'innovazione in diabetologia è guidato anche questa volta dal piede diabetico. I due case report presentati sono particolarmente interessanti, esplorano infatti trattamenti non ancora abituali ed esperienze che aprono nuove prospettive terapeutiche.

L'articolo di Graziani affronta una condizione di estrema difficoltà come quella del trattamento delle arteriopatie multicomplicate nel paziente diabetico, condizioni che spesso esitano in amputazioni maggiori. Il rivaroxaban a basso dosaggio, in associazione alla terapia antiaggregante piastrinica, si propone come trattamento dei nostri pazienti ad alto rischio trombotico.

L'articolo di Stroffolini affronta invece il problema delle osteomieliti da Gram+ e, nel caso di una scelta il più possibile conservativa, il trattamento antibiotico prolungato. Anche in questo caso un antibiotico long-acting come la dalbavancina può essere utile per ridurre tempi di ricovero e i costi di gestione della terapia, quanto mai preziosi in questo periodo, e favorire esiti positivi nel rispetto della stewardship.

Che dire... penso che non sia possibile rinunciare ai rapporti umani cosi come li conosciamo e nell'attesa di ritornare ad abbracciarci, continuiamo a narrare. Buona lettura, buona estate, a presto. 